\title{
BMJ Open Impact and longevity of measles- associated immune suppression: a matched cohort study using data from the THIN general practice database in the UK
}

\author{
Kartini Gadroen, ${ }^{1}$ Caitlin N Dodd, ${ }^{1}$ Gwen M C Masclee, ${ }^{1}$ Maria A J de Ridder, ${ }^{1}$ \\ Daniel Weibel, ${ }^{1}$ Michael J Mina, ${ }^{2}$ Bryan T Grenfell, ${ }^{3}$ Miriam C J M Sturkenboom, ${ }^{1}$ \\ David A M C van de Vijver, ${ }^{4}$ Rik $L$ de Swart ${ }^{4}$
}

To cite: Gadroen K, Dodd CN, Masclee GMC, et al. Impact and longevity of measles-associated immune suppression: a matched cohort study using data from the THIN general practice database in the UK. BMJ Open 2018:8:e021465. doi:10.1136/ bmjopen-2017-021465

- Prepublication history and additional material for this paper are available online. To view these files, please visit the journal online (http://dx.doi. org/10.1136/bmjopen-2017021465).

DAMCV and RLS contributed equally.

$\mathrm{KG}$ and CND contributed equally.

Received 3 January 2018 Revised 18 September 2018 Accepted 27 September 2018

Check for updates

(C) Author(s) (or their employer(s)) 2018. Re-use permitted under CC BY-NC. No commercial re-use. See rights and permissions. Published by BMJ.

For numbered affiliations see end of article.

Correspondence to

Dr Rik L de Swart;

r.deswart@erasmusmc.nl

\section{ABSTRACT}

Objective To test the hypothesis that measles infection increases the incidence of non-measles infectious diseases over a prolonged period of time.

Design A population-based matched cohort study. Data sources This study examined children aged 1-15 years in The Health Improvement Network UK general practice medical records database. Participants included 2228 patients diagnosed with measles between 1990 and 2014, which were matched on age, sex, general practitioner practice and calendar year with 19930 children without measles. All controls had received at least one measles vaccination. Children with a history of immune-compromising conditions or with immunesuppressive treatment were excluded.

Primary outcome measures Incidence rate ratio (IRR) of infections, anti-infective prescriptions and all-cause hospitalisations following measles in predetermined periods using multivariate analysis to adjust for confounding variables.

Results In children with measles, the incidence rate for non-measles infectious disease was significantly increased in each time period assessed up to 5 years postmeasles: $43 \%$ in the first month (IRR: $1.43 ; 95 \% \mathrm{Cl}$ 1.22 to 1.68 ), $22 \%$ from month one to the first year (IRR: $1.22 ; 95 \% \mathrm{Cl} 1.14$ to 1.31 ), $10 \%$ from year 1 to 2.5 years (IRR: $1.10 ; 95 \% \mathrm{Cl} 1.02$ to 1.19 ) and $15 \%$ (IRR: 1.15; $95 \% \mathrm{Cl} 1.06$ to 1.25 ) in years 2.5 to 5 years of followup. Children with measles were more than three times as likely to receive an anti-infective prescription in the first month and $15 \%-24 \%$ more likely between the first month and 5 years. The rate of hospitalisation in children with measles was increased only in the month following diagnosis but not thereafter (IRR: $2.83 ; 95 \% \mathrm{Cl} 1.72$ to 4.67).

Conclusion Following measles, children had increased rates of diagnosed infections, requiring increased prescribing of antimicrobial therapies. This populationbased matched cohort study supports the hypothesis that measles has a prolonged impact on host resistance to non-measles infectious diseases.

\section{Strength and limitations of this study}

- Strength: cohort represents the full range of patients seen in routine clinical practice in a high-income country.

- Strength: the matched study design allowed estimation of the effect of measles on non-measles infections and anti-infective prescriptions over a prolonged period of time.

- Limitation: a key assumption of the study method is that children with measles and children free of measles are comparable. Although major confounders were adjusted for, there could potentially be residual confounding.

- Limitation: the cohort was assembled according to the Read diagnosis code for 'measles' from the electronic medical record. Laboratory confirmation is lacking for most of the identified cases. Validation studies to identify measles and assess the accuracy of the date of diagnosis using this type of database are also lacking. As a result, some patients may have been misclassified.

\section{INTRODUCTION}

Measles is a highly contagious childhood disease. ${ }^{1}$ During the prevaccine era, nearly every child acquired measles before the age of 15 years. ${ }^{2}$ A key characteristic of the disease is a transient immune suppression, causing increased susceptibility to opportunistic infections. As a result, measles is often complicated by pneumonia, diarrhoea or otitis media, which may lead to severe and even fatal disease. ${ }^{3} 4$ The introduction of measles-containing vaccines has reduced measles incidence ${ }^{1}$ as well as childhood mortality. ${ }^{5}$ Interestingly, this reduction in childhood mortality is stronger than what would have been expected based on measles mortality in unvaccinated populations. ${ }^{6}$ 
Although measles virus is transmitted via the respiratory route, it predominantly infects immune cells and causes systemic disease. ${ }^{17}$ Recent studies into the mechanism of measles immune suppression, based on observations in experimentally infected non-human primates, showed that measles virus preferentially replicates in $\mathrm{CD} 150^{+}$ memory lymphocytes. ${ }^{8-10}$ It was hypothesised that viral cytotoxicity and immune-mediated clearance resulted in depletion of these cells, leading to a loss of acquired immunological memory. ${ }^{49}$ Consistent with this hypothesis, a subsequent ecological study using population level data from England and Wales, the USA and Denmark found that rates of non-measles infectious disease mortality are tightly coupled to measles incidence-with a greater mortality rate at higher recent measles incidence. Mina et al measured a duration of measles-induced immunomodulation by assessing the association between measles incidence and childhood mortality. The results showed that measles was associated with increased mortality from other infectious diseases over a period of more than 2 years. ${ }^{11}$ However, the study was based on population-level ecological association data, and the authors did not have access to case-based data.

Monovalent measles vaccination was introduced in England in 1968 and replaced in 1988 by the multivalent measles, mumps and rubella (MMR) vaccine. Initially, MMR was offered only as a single dose at the age of 12 months. In 1996, a second dose was introduced and offered at age of 40 months. From 1996 to 2004, the number of reported measles cases in the UK was small. Following the publication of a subsequently discredited study linking autism and measles vaccination in 1998, coverage dropped for several years below herd protection level, and in 2007, measles was re-established in the UK. In response, an MMR catch-up campaign targeting individuals up to 18 years of age was implemented in 2008. In response to a mumps outbreak, Wales had already implemented a national MMR vaccination campaign targeting individuals aged between 11 years and 25 years in 2005 .

In the present study, we have used individual-level data from a UK database to test whether measles results in prolonged increased susceptibility to other infections. The aim of our study was to assess whether measles is associated with increased frequency of non-measles infectious disease, anti-infective prescriptions or hospitalisations over a prolonged period of time.

\section{METHODS}

\section{Data source}

For this matched-cohort study, we used data from The Health Improvement Network (THIN) database. THIN is a population-based general practice registry that contains prospectively collected, anonymised longitudinal electronic patient records from over 550 general practitioner (GP) practices across the UK, capturing healthcare data from more than 12 million patients (about $6 \%$ of the population). ${ }^{12} 13$ Data recorded in THIN include demographic, socioeconomic and clinical information, including chief complaint, symptoms, test results, diagnoses, prescriptions and referrals to hospitals. The population covered has similar demographic characteristics to the national UK population, and the recording of consultations and prescriptions is comparable with national levels. ${ }^{1415}$ Diagnoses and symptoms are recorded in Read codes, a standard terminology, maintained by the UK National Health Service Centre for Coding and Classification. ${ }^{16}$ Information on drug prescription is recorded using British National Formulary codes and the MULTILEX product dictionary. The specific codes used for this study were selected by a medical doctor and reviewed by a virologist, medical doctor and epidemiologist for their relevance (see online supplementary file S1 for selected read codes).

\section{Study design and population}

The source population consisted of all patients who had contributed longitudinal data to the database between 1 January 1990 to 30 September 2014, from the age of 6 months to 15 years. This study period captures the period of time when vaccination rates fell during the late 1990s, with increased measles cases in the following years. The measles group consisted of children with a measles diagnosis (whether or not laboratory confirmed) between the ages of 1 year and 15 years. The date of measles diagnosis was taken as the index date. To each child with a measles diagnosis, up to 10 children free of measles were matched on age in years, sex, GP practice and calendar time in years. Children free of measles were required to have had at least one dose of measles-containing vaccine, prior to the matched case's index date. We considered that having received at least one dose of measles-containing vaccine would reduce the chance that children included in the 'free of measles' group had ever had measles. Patients with a history of immune-compromising conditions (eg, HIV infection and organ or bone marrow transplantation), or with immune suppressive treatment prior to the index date were excluded (see supplementary file S2 Table for the STROBE statement of this study).

\section{Patient involvement}

No patient was involved in setting the research question, outcome measures, design or conduct of the study. The results were not disseminated to the patients, as the study was based on anonymised patient records.

\section{Outcomes}

Three clinical outcomes were considered: infections, anti-infective prescriptions and all-cause hospitalisations. The outcomes were defined by the relevant clinical codes for symptoms and diagnoses or drug codes. Infections included all communicable diseases other than measles. Infections were required to be 14 days apart to be considered a new event. Anti-infective prescriptions included all systemic antibiotics, antimycotic, antivirals and antiparasitic medication. For anti-infective prescriptions and 


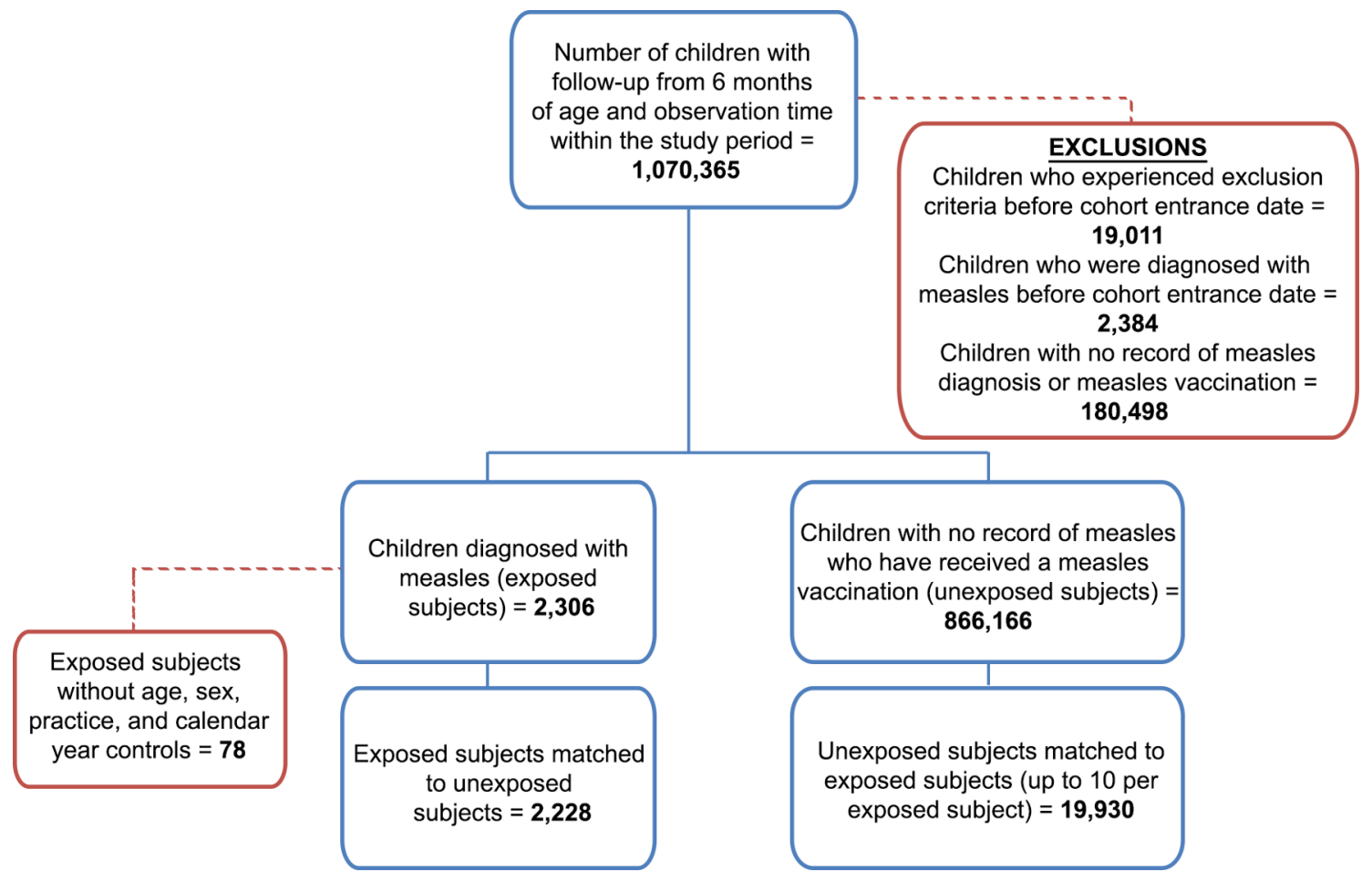

Figure 1 Flow chart of study cohort selection. Starting from 1070365 eligible children in the THIN database, 2228 measles patients and 19930 matched controls were selected for this study. THIN, The Health Improvement Network.

hospitalisations, any event occurring on a different day (at least 1 day apart) was considered a new event.

\section{Follow-up}

Follow-up started at the index date and continued for a period of 5 years, until date of transfer out the GP's practice, the 15th birthday or death, whichever came earliest. Each outcome was analysed in predetermined periods following measles diagnosis: within the first month; $\geq 1$ month to $<1$ year; $\geq 1$ year to $<2.5$ years; and $\geq 2.5$ years to $<5$ years, to observe changes over time. HRs for hospitalisation were calculated with follow-up starting at 30 days after the index date to avoid inclusion of hospitalisations due to initial complications related to measles.

\section{Potential confounders and effect modifiers}

We considered as potential confounders: chronic respiratory disease, cardiovascular disease, prior exposure to routine childhood vaccines other than measles containing vaccines, deprivation index, healthcare consumption and occurrence of each outcome of interest in the year prior to index. Potential confounders were assessed at the index date. Vaccine adherence was defined as exposure to any dose of other routine childhood vaccines such as pertussis-containing vaccines before the index date and coded as binary with vaccine adherence equal to one if any other childhood vaccine was received and zero otherwise. The Townsend deprivation score, a measure of social deprivation based on unemployment level, car ownership, home ownership and household overcrowding levels by area, was used within a particular zip code. ${ }^{17}$ Healthcare consumption, as a proxy for general health, was assessed by the rate of GP consultations in the year prior to the index date ${ }^{18}$ and categorised using quintile cut-off points. For a list of various types of consultations included to calculate GP consultation rate, see online supplementary file S3. For each outcome, the event rate in the year prior to index was calculated.

\section{Statistical analysis}

Baseline characteristics were compared between children with measles and children free of measles using Student's t-test, Mann-Whitney U test, $\chi^{2}$ test or Fisher's exact test as appropriate. Observed incidence rates of measles diagnosis codes as well as measles notification codes were estimated by dividing the number of cases by the number of person-years at risk within the database stratified by calendar year and were compared with expected incidence rates, derived from publicly available official statistics from the UK National Archives. ${ }^{19}$ The differences in incidence of the outcomes between children with measles and children free of measles were analysed for each period using Poisson regression. For this analysis, matching was relaxed due to uninformative matched strata for each outcome, with over 1000 uninformative strata for the hospitalisation outcome. A stratified analysis was therefore not conducted, and the analysis was adjusted for confounding using multivariable analysis. We submitted the following confounders: history of cardiovascular malformation, history of respiratory disease, exposure to childhood vaccinations other than measles containing vaccination, age, sex and GP consultation rate. Exposure to childhood vaccinations other 
Table 1 Baseline characteristics of enrolled subjects

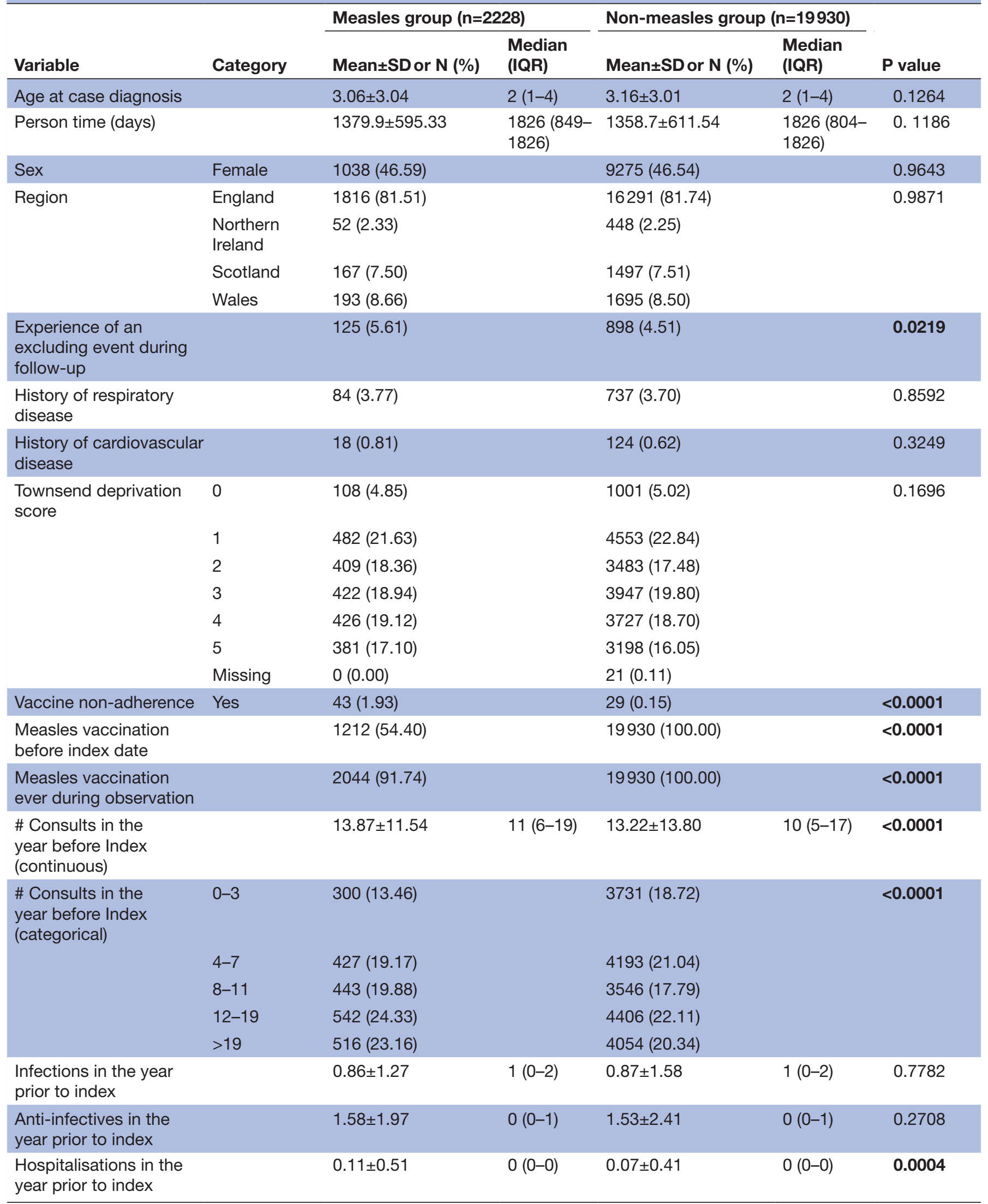

$P$ values lower than 0.05 are shown in bold. 


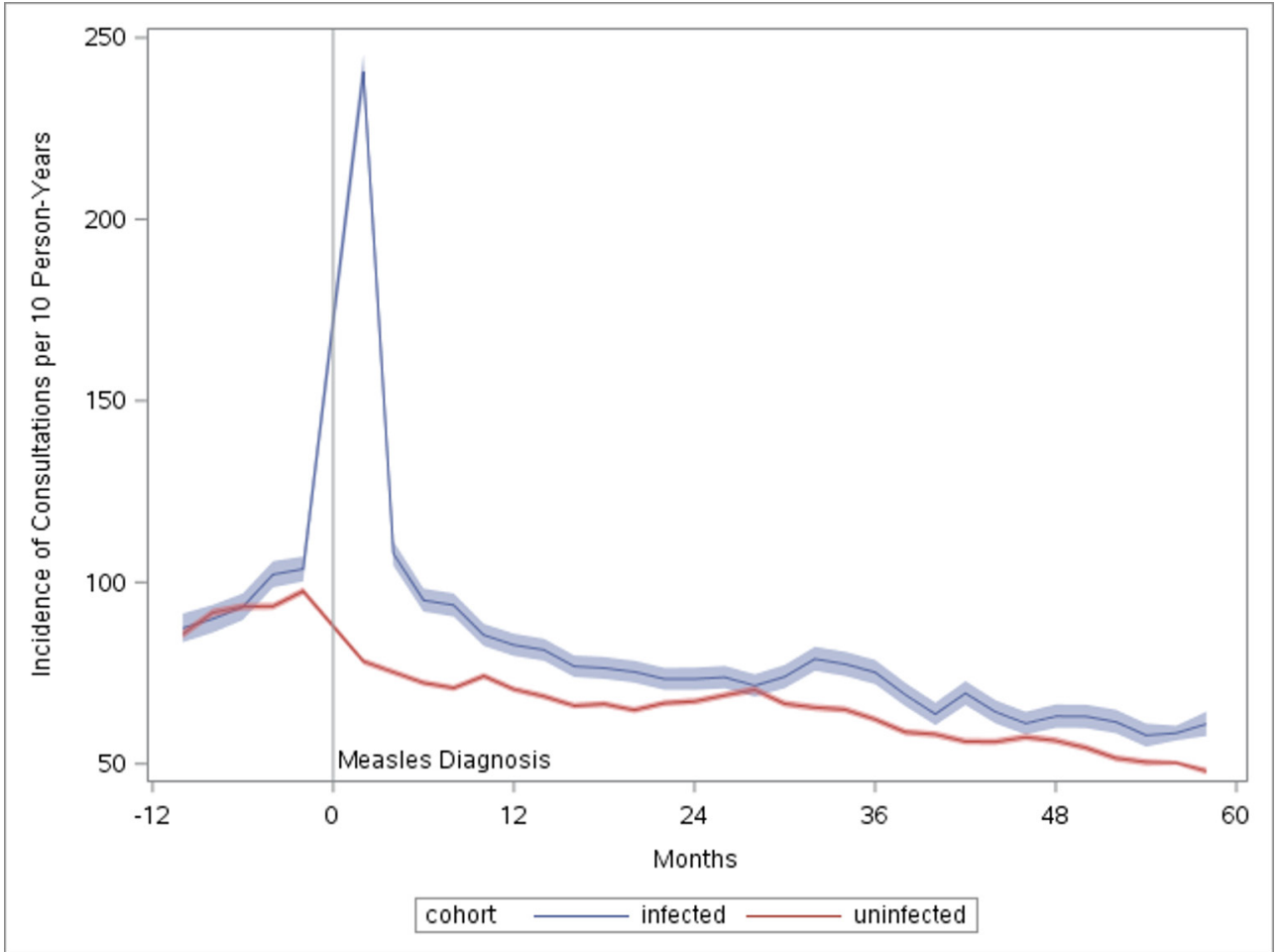

Figure 2 Consultations in measles patients and matched controls. Incidence rates of consultations in children diagnosed with measles (blue lines) or matched controls (red lines) per 10 person-years, plotted by time (in months) before or after diagnosis of measles. The vertical dotted line indicates the time point of diagnosis in the measles patients. The shaded areas represent $95 \%$ Cls.

than measles-containing vaccination was not retained in final models. In addition, per outcome, we submitted rate of the outcome in the year prior to the index date. Absolute rates of each outcome per 1000 person-days were calculated with covariates fixed as follows: cardiovascular and respiratory history=no; receipt of other childhood vaccines=yes; number of consults and events in the previous year $=$ median; age $=3$ years; and $s e x=f e-$ male. Kaplan-Meier curves and log-rank tests were used to compare time with first hospitalisation between measles infected and control individuals, with follow-up beginning at 30 days after the index date (to avoid including codes related to the initial measles infection). A stratified Cox proportional hazards model, stratified by matched set and adjusted for confounding variables, was applied to estimate HRs comparing children with measles and children free of measles. Assumptions of proportional hazards were assessed by inspecting the Kaplan-Meier curves and formally tested with inspection of a measles*time interaction term. Model selection was by backward covariate selection, with the criteria $\mathrm{p}<0.1$. Subsequently, we verified automatically selected models using minimisation of Akaike's Information Criterion (AIC). We also estimated the HRs for the outcomes first infection and first prescription.

\section{Sensitivity analysis}

Children who have received vaccinations may be different in their underlying health status, social background, lifestyle, healthcare-seeking behaviour and healthcare utilisation from those who did not receive vaccinations. To examine the possible effect of these unmeasured confounders, we conducted a sensitivity analysis, stratifying the data into matched sets in which all measles cases had received (ie, non-measles group vaccinated vs measles group vaccinated) or had not received a measles-containing vaccine (ie, non-measles group vaccinated vs measles-group unvaccinated). In post hoc analyses, we assessed the incidence rate ratio (IRR) of each outcome over the entire study period in vaccine adherent versus non-adherent children for each outcome using Poisson regression. We also examined the correlation of the consultation rate the year before and after the index date in measles versus control groups using linear regression. For data management and analysis, we used SAS V.9.3.

\section{RESULTS}

From the database population of 1070365 children aged 1-15 years, we identified 2228 eligible children with a measles diagnosis. These children were matched to 19930 children free of measles. Figure 1 illustrates the composition of the study cohort. Table 1 describes baseline characteristics of children with measles and children free of measles. Median follow-up time was 5.0 years (IQR: 2.2-5.0). The incidence rate of measles and of measles notification as reported in the THIN database were similar to the expected overall confirmed measles incidence rate as reported by official UK Government 
Table 2 Descriptive statistics of events in enrolled measles exposed and measles-non exposed children

\begin{tabular}{|c|c|c|c|c|c|c|}
\hline \multirow[b]{2}{*}{ Variable } & \multirow[b]{2}{*}{ Category } & \multicolumn{2}{|c|}{ Measles group $(n=2228)$} & \multicolumn{2}{|c|}{ Non-measles group $(n=19930)$} & \multirow[b]{2}{*}{$P$ values } \\
\hline & & Mean \pm SD or N (\%) & $\begin{array}{l}\text { Median } \\
\text { (IQR) }\end{array}$ & Mean \pm SD or N (\%) & $\begin{array}{l}\text { Median } \\
\text { (IQR) }\end{array}$ & \\
\hline \# Infections (continuous) & & 1. $61 \pm 2.17$ & $1(0-2)$ & $1.28 \pm 1.85$ & $1(0-2)$ & $<0.0001$ \\
\hline \multirow[t]{5}{*}{ \# Infections (categorical) } & 0 & $864(38.78)$ & & $9224(46.28)$ & & $<0.0001$ \\
\hline & $1-2$ & $856(38.42)$ & & $7184(36.05)$ & & \\
\hline & $3-5$ & 377 (16.92) & & $2852(14.31)$ & & \\
\hline & $6-10$ & $115(5.16)$ & & $591(2.97)$ & & \\
\hline & $>10$ & $16(0.72)$ & & $79(0.40)$ & & \\
\hline $\begin{array}{l}\text { \# Anti-infective Rx } \\
\text { (continuous) }\end{array}$ & & $4.58 \pm 5.45$ & $3(1-6)$ & $3.35 \pm 4.43$ & $2(0-5)$ & $<0.0001$ \\
\hline \multirow{6}{*}{$\begin{array}{l}\text { \# Anti-infective Rx } \\
\text { (categorical) }\end{array}$} & 0 & $326(14.63)$ & & $5104(25.61)$ & & $<0.0001$ \\
\hline & $1-2$ & $631(28.32)$ & & $6168(30.95)$ & & \\
\hline & $3-5$ & $651(29.22)$ & & $4617(23.17)$ & & \\
\hline & $6-10$ & $393(17.64)$ & & $2892(14.51)$ & & \\
\hline & $11-20$ & $187(8.39)$ & & $979(4.91)$ & & \\
\hline & $>20$ & $40(1.80)$ & & $170(0.85)$ & & \\
\hline $\begin{array}{l}\text { \# Hospitalisations } \\
\text { (continuous) }\end{array}$ & & $0.16 \pm 0.74$ & $0(0-0)$ & $0.12 \pm 0.63$ & $0(0-0)$ & 0.0001 \\
\hline \multirow{5}{*}{$\begin{array}{l}\text { \# Hospitalisations } \\
\text { (categorical) }\end{array}$} & 0 & 1999 (89.72) & & $18369(92.17)$ & & 0.0014 \\
\hline & $1-2$ & $204(9.16)$ & & $1396(7.00)$ & & \\
\hline & $3-5$ & $20(0.90)$ & & $134(0.67)$ & & \\
\hline & $6-10$ & $3(0.13)$ & & $24(0.12)$ & & \\
\hline & $>10$ & $2(0.09)$ & & $7(0.04)$ & & \\
\hline
\end{tabular}

$\mathrm{Rx}$, drug treatment.

statistics (see supplementary file S4 figure). However, between 1999 and 2006 diagnoses in THIN decrease while notifications and the population incidence increase. There was no significant difference in follow-up time between the children with measles and the children free of measles. Exposure to childhood vaccines other than measles-containing vaccines prior to the index date was lower among children with measles $(98.1 \%$ vs $99.8 \%)$, but this difference was small compared with the difference in vaccination coverage of measles-containing vaccines prior to the index date $54.4 \%$ in children with measles vs $100 \%$ in children free of measles, due to inclusion criteria). GP consultation rate in the year prior to index date was slightly higher in the measles group than in the non-measles group: mean 13.87 versus 13.22 ( $p<0.001)$ consults in the year prior, respectively (figure 2 ). The Townsend deprivation index was similar in children with measles and children free of measles. The rate of infections and anti-infective prescriptions prior to index were similar between measles and non-measles subjects, while hospitalisations prior to index were more frequent for subjects subsequently diagnosed with measles. Table 2 describes events of interest occurring during follow-up in measles and non-measles subjects.

\section{Infectious disease}

The most frequently occurring infectious diseases were upper respiratory infectious diseases (for details see online Supplementary file S5 table). The IRR (table 3) of infections for children with measles compared with children free of measles was $43 \%$ higher in the first month (IRR: $1.43 ; 95 \%$ CI 1.22 to 1.68 ), 22\% higher from the first month to the first year (IRR: 1.22; 95\% CI 1.14 to 1.31 ), $10 \%$ higher from the first year to 2.5 years (IRR: 1.10 ; 95\% CI 1.02 to 1.19 ) and $15 \%$ higher (IRR: 1.15 ; 95\% CI 1.06 to 1.25 ) in the $2.5-5$ years of follow-up (figure 3 ).

The absolute rate of infections per 1000 person-days in the first month to first year was 1.7 (95\% CI 1.6 to 1.9) for children with measles and 1.33 (95\% CI 1.29 to 1.36) for children free of measles. The adjusted HR for non-measles infectious disease over the full follow-up period starting 30 days after measles diagnosis was 1.20 (95\% CI 1.13 to 1.28 ) (see online Supplementary file S6).

\section{Prescriptions}

Children with measles received more anti-infective prescriptions than children without measles in all periods (table 3, figure 4, online Supplementary file S5 and Supplementary file S7). The absolute rate of 
Table 3 Incidence rate ratios (IRRs) of events of interest in predefined time periods following measles infection

IRR (95\% CI)

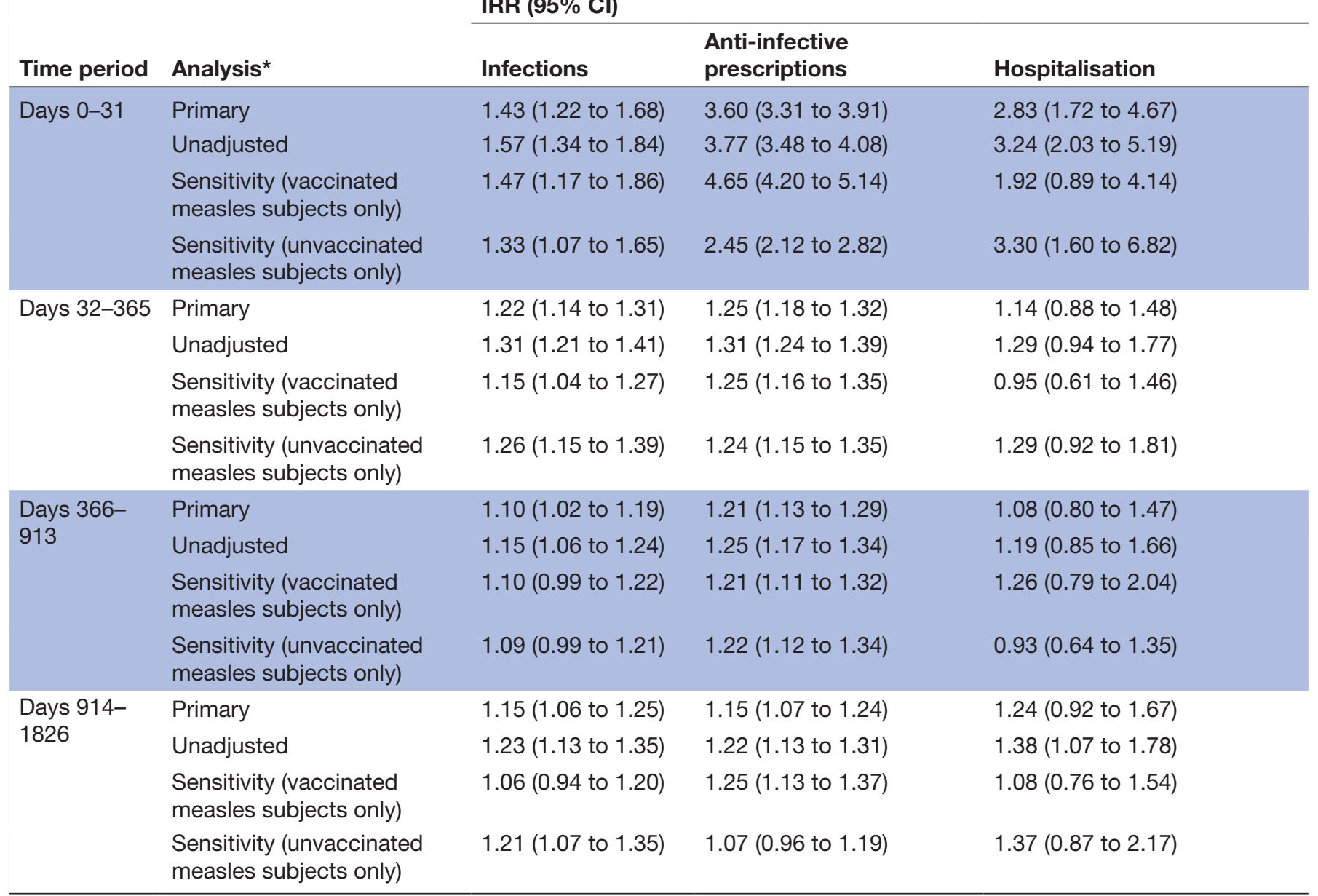

${ }^{*}$ Primary and sensitivity analyses were adjusted for: frequency of consultations in the year prior to index, frequency of the outcome of interest in the year prior to index, history of cardiovascular malformation, history of respiratory disease, age and sex.

anti-infective prescriptions per 1000 person-days in the first month to first year was 0.55 (95\% CI 0.51 to 0.59$)$ for children with measles and 0.45 (95\% CI 0.43 to 0.47$)$ for children free of measles. The adjusted HR for anti-infective prescription over the full follow-up period starting 30 days after measles diagnosis was 1.24 (95\% CI 1.18 to 1.31). Within the first month of follow-up, children with measles had more than a threefold increase in use of anti-infective drugs as compared with controls (IRR: 3.60; $95 \%$ CI 3.31 to 3.91 ). Following the first month, children who had measles continued to use more anti-infective drugs over the entire duration of the follow-up: 1 month-1 year (IRR 1.24; 95\% CI 1.18 to 1.32 ); 1 year2.5 years (IRR $1.21 ; 95 \%$ CI 1.13 to 1.29 ) and 2.5 years -5 years (IRR $1.15 ; 95 \%$ CI 1.07 to 1.24 ).

\section{Hospitalisation}

Despite smaller sample sizes, the analysis on hospitalisations also showed increased IRRs, although these were significant during the first period only (figure 5). In the Cox proportional hazards model, confounder selection using either backward selection, or minimisation of the AIC resulted in the same model, namely control for the hospitalisation rate prior to the index date, the GP consultation rate in the year prior to index date and history of cardiac malformation. The absolute rate of hospitalisations per 1000 person days in the first month to first year was equal at $0.2(95 \%$ CI 0.1 to 0.2$)$ for children with measles and children free of measles. The adjusted HR of hospitalisation for measles versus non-measles subjects was 1.12 (95\% CI 0.96 to 1.31 ).

\section{Sensitivity analysis}

Results of the sensitivity analysis were partially in agreement with findings from the main analysis. When we restricted the analysis to only those children who had received measles vaccination prior to receiving a diagnosis of measles $(54.4 \%$ of all eligible children with a measles diagnosis), differences to the main analyses were not observed for anti-infective prescriptions. However, an increased rate of hospitalisations was no longer detected in any time period and an increased rate of infections no longer extended beyond 1year postdiagnosis. In the analysis restricted to those children who had not had a measles vaccination prior to receiving a diagnosis of measles $(45.6 \%$ of all eligible children with a measles diagnosis), the results were in line with the main findings for hospitalisations, infections and anti-infective prescriptions 


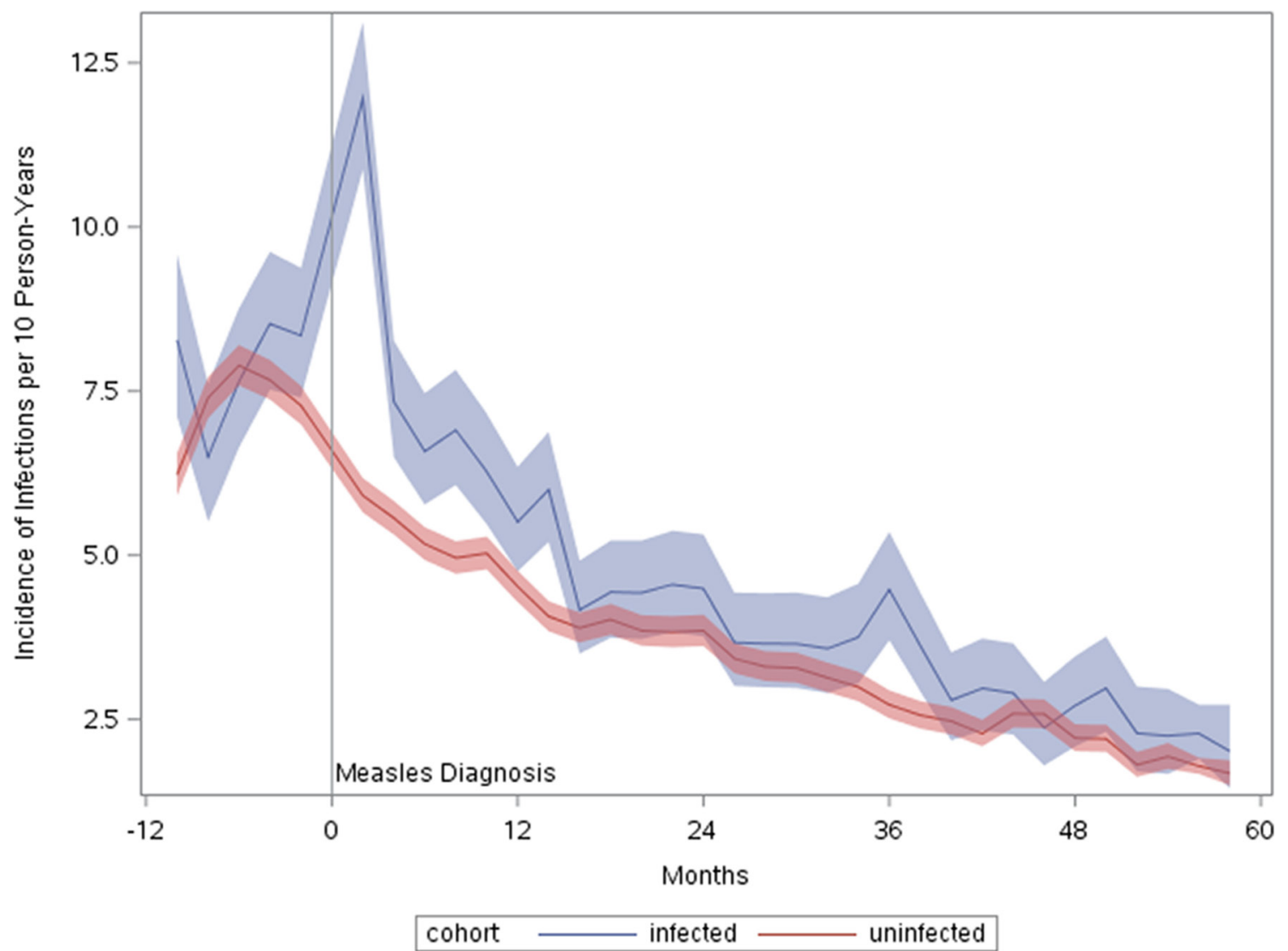

Figure 3 | Infections in measles patients and matched controls. Incidence rates of infections in children diagnosed with measles (blue lines) or matched controls (red lines) per 10 person-years, plotted by time (in months) before or after diagnosis of measles. The vertical dotted line indicates the time point of diagnosis in the measles patients. The shaded areas represent $95 \%$ Cls.

with the exception that increased risk for anti-infective prescriptions did not extend into the period $2.5-5$ years following measles.

Post hoc analysis of the impact of vaccine adherence regardless of measles status revealed that vaccine non-adherent children were $42 \%$ more likely to receive an anti-infective prescription than vaccine-adherent children. There was no difference in risk of infections or hospitalisations. Regressing postindex consults on preindex consults and measles, or non-measles status revealed that both groups showed similar trends with the rate of consultation before index date higher than that after the index date.

\section{DISCUSSION}

To our knowledge, this is the first matched-cohort study to investigate the longevity of measles-associated immune suppression in a high-income country. The results of this study are in strong agreement with previous non-clinical and ecological studies also in high-income countries. ${ }^{11}$ We found that rates of diagnosed infections and anti-infective prescriptions are elevated following measles infection for up to 5 years. While increased risk of infections and anti-infective prescriptions remained statistically significant over the full 5-year study period, the effect size diminished particularly after the first year, and statistical significance is partly explained by our large sample size. Children diagnosed with measles were hospitalised more frequently than children free of measles, although this was only significant in the first month following infection. When we excluded the first month postmeasles, the time to first hospitalisation did not differ between the measles group and the non-measles group. This could be explained, at least in part, by a survival bias, whereby a disproportionately large number of measles cases entered the hospital during the first month, and these may have represented the most severe cases. Additionally, a lack of effect on hospitalisation after the first month was likely a result of the low overall number of hospitalisations in our cases and controls. We acknowledge that the first interval spanning 1 month-1year postmeasles is wide and have conducted analysis using smaller intervals, the results of which can be found in the supplementary material (see online Supplementary file S8 table). These results are in agreement with our primary analysis, with risk of infections and anti-infectives remaining elevated throughout the entirety of the first year following measles and risk of hospitalisations elevated in the first month following measles only.

The incidence rates of infections, anti-infective prescriptions and hospitalisations in the measles group appear to 


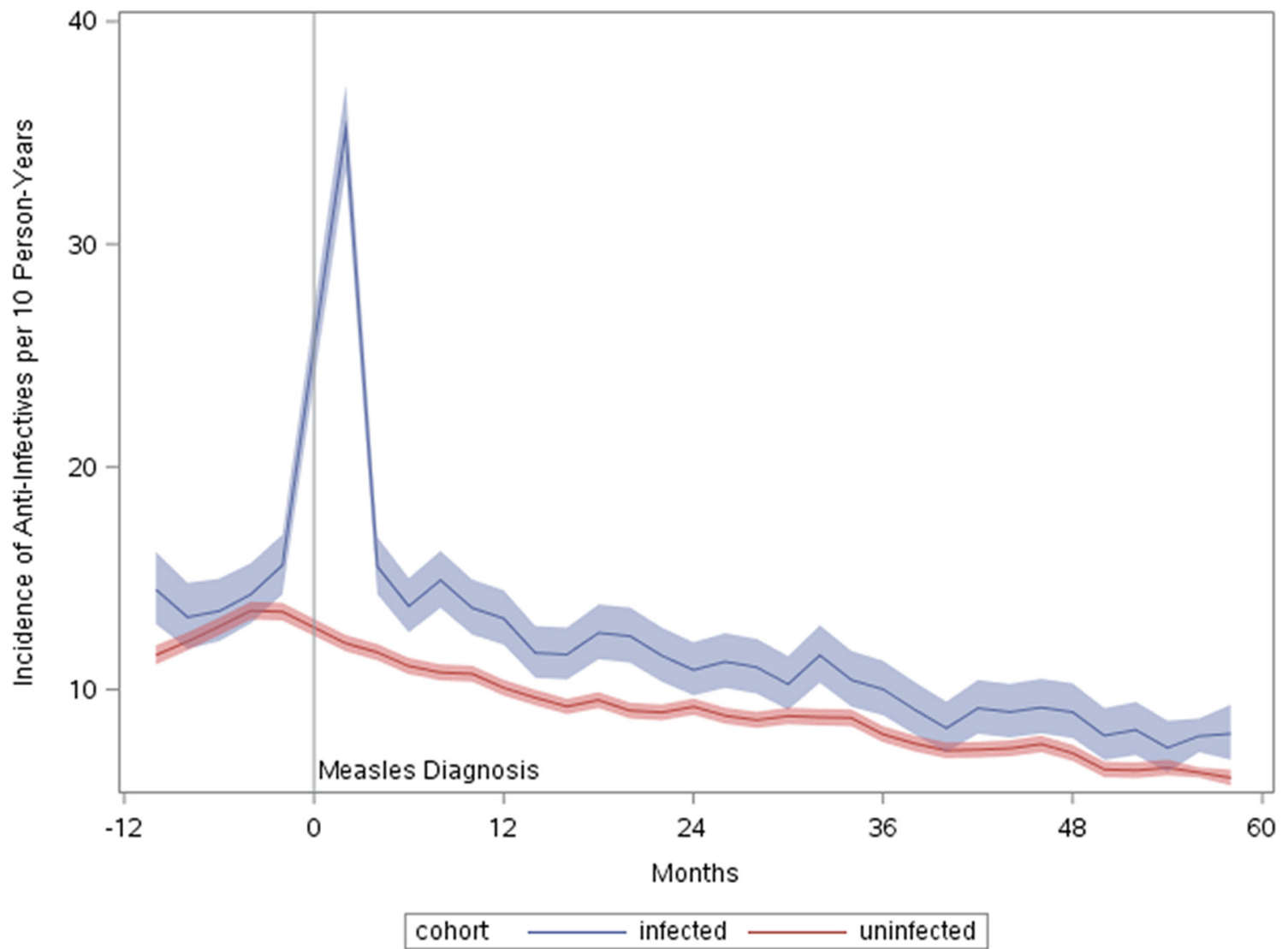

Figure 4 Anti-infective prescriptions in measles patients and matched controls. Incidence rates of anti-infective prescriptions in children diagnosed with measles (blue lines) or matched controls (red lines) per 10 person-years, plotted by time (in months) before or after diagnosis of measles. The vertical dotted line indicates the time point of diagnosis in the measles patients. The shaded areas represent $95 \%$ Cls.

increase prior to the index date, that is, before they got measles (figures 3-5). This could partially be explained by a lag time between a suspected diagnosis and a definite diagnosis. In some instances, a GP may have coded a definite diagnosis on the date a confirmation had been received either from the lab or from the hospital. For some outcomes, however, the rise in incidence begins months before diagnosis. Validation studies to assess the accuracy of the date of diagnosis using this type of database are lacking.

To be considered a new event, prescriptions only had to be given on a different day. Acknowledging that a prescription can be changed if there is poor response or allergy to the first drug, we also examined the effect of anti-infective prescription, considering a 14-day interval between anti-infective prescriptions. This did not change the significance or direction of any result (results not shown). Both groups revealed similar trends with the rate of consultation before index date higher than after index date. ${ }^{20} 21$ This is most likely related to age. Although measles is a statutory notifiable infectious disease under EU legislation, ${ }^{22}$ an under-reporting of (severe) cases, who might have by-passed the GP and gone directly to the hospital, cannot be ruled out. Also, it is possible that a mild measles infection would not have prompted a visit to the GP and may have gone undetected as well. ${ }^{2324}$ This means that we may have missed some children with measles. It should be noted though that laboratory confirmation for most of the identified cases is lacking. As a result, some patients may have been misclassified. Validation studies to accurately identify measles using this type of database are also lacking. In case of non-differential misclassification, the direction of the bias is likely to be towards the null value, so one would expect to see a larger estimate if misclassification was absent. Differential misclassification however can inflate or attenuate the effect estimates. To minimise the impact of differential misclassification, we examined the consultation rate in both measles and non-measles groups. To provide additional assurance that controls were children truly free of measles, controls had to have at least one measles-containing vaccination prior to the index date. An advantage of this type of observational study is that it is not necessary to identify all outcomes in all children in order to obtain an unbiased estimate. A key assumption, however, is comparability of children with measles and children free of measles. In order to ensure that the children with measles and the children free of measles were comparable, we matched them on confounding factors such as age, sex, GP practice and calendar time. We also considered including experiencing an excluding event (ie, an immune-compromising condition or immune suppressive treatment) as a censoring 


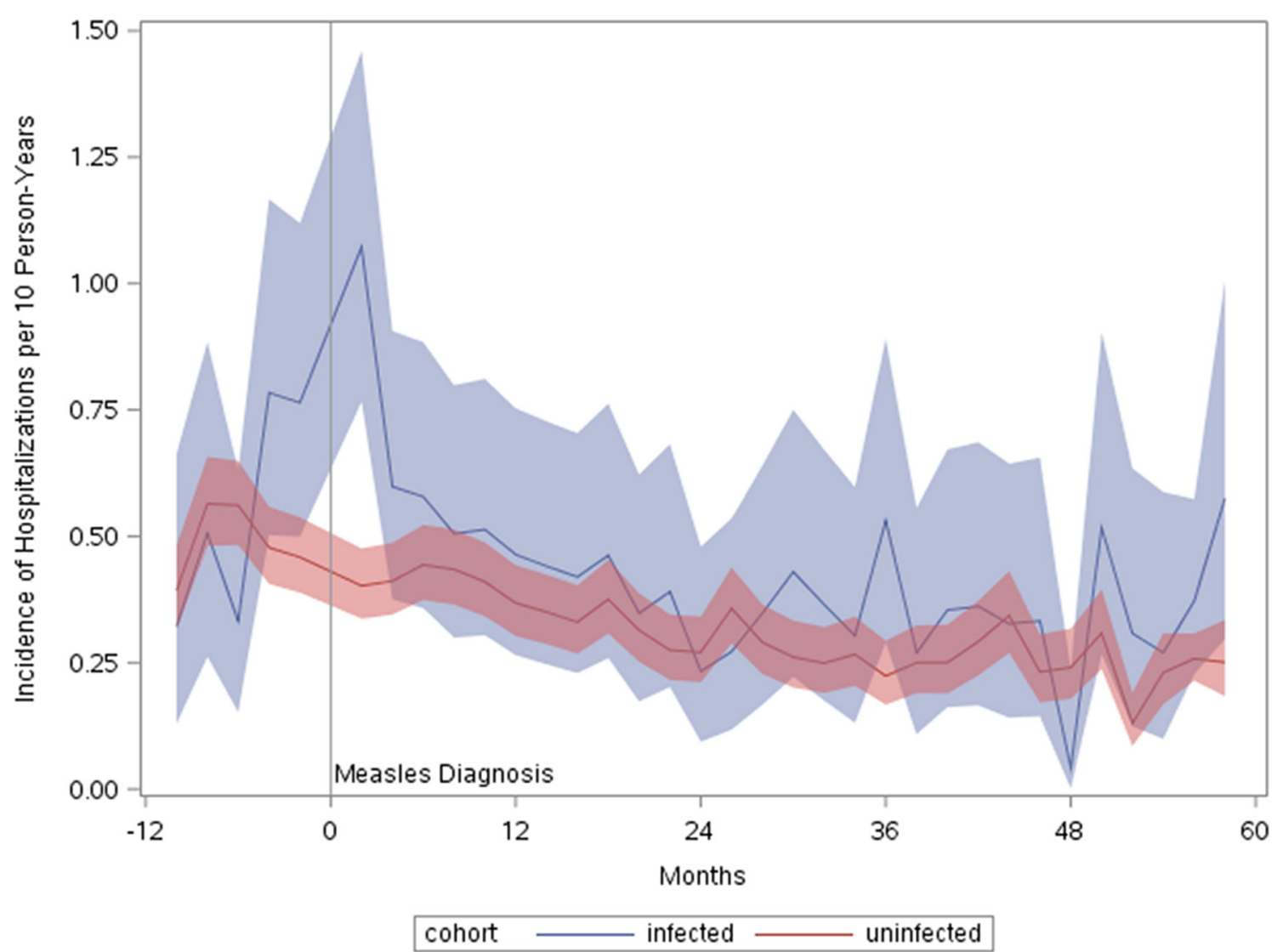

Figure 5 | Hospitalisations in measles patients and matched controls. Incidence rates of hospitalisations in children diagnosed with measles (blue lines) or matched controls (red lines) per 10 person-years, plotted by time (in months) before or after diagnosis of measles. The vertical dotted line indicates the time point of diagnosis in the measles patients. The shaded areas represent $95 \%$ Cls.

variable but determined this was not consistent with our matching strategy: the groups were matched to be comparable at index. Nevertheless, we acknowledge that it is possible that confounding due to differences in underlying health status, social background, lifestyle, health seeking behaviour and healthcare utilisation between children with measles and children free of measles may have occurred. The complexity of these factors makes them difficult to control. We attempted to overcome the confounding effect of underlying health status by excluding children with a history of immune-compromising conditions, and controlling for comorbidities such as cardiovascular disease, and respiratory disease. We assessed social background and lifestyle by testing for differences in social deprivation within a particular zip code and matching on practice. Certain children may have had a lower threshold for visiting the GP and therefore may have had a higher likelihood of receiving a diagnosis of measles (particular during an outbreak) and may also have been diagnosed more frequently with other infectious diseases and/or may have received a prescription for anti-infectives more frequently. To investigate this, we included GP consultation rate in the year prior to cohort entry as a covariate in each of our models. In the unmatched Poisson analyses, we did not control for all potential confounders. Because 472 unique practices were represented in the cohort, it was impossible to control for practice. Similarly, the 25 years included in the study period make control for calendar year infeasible unless calendar year is treated as a continuous variable, which would require the assumption of a linear relationship between year and $\log$ (events). To address the potential effect of calendar time, we have conducted analyses stratified by calendar period (before 2005 and after 2004) and included these results in supplementary material (see online Supplementary file S9 table).

Because vaccinated and unvaccinated children may differ in their health-seeking behaviour or likelihood of acquiring infectious disease, we conducted a sensitivity analysis in two strata: (1) restricting to only those children who had received a measles vaccination prior to the index date and (2) restricting to only those children who were unexposed to measles vaccination prior to the index date. Results from both subanalyses were in line with the findings from the main analysis with the exceptions that the period of increased risk for infections did not extend past 1 year and no increased risk for hospitalisations was detected when analysis was limited to measles-vaccinated children.

We did not adjust for measles vaccination after index date in the context of postexposure prophylaxis because many exposed persons are not identified until more 
than 72 hours after initial exposure, which is too late for prophylaxis with measles vaccine. ${ }^{2}$ Post hoc analysis of vaccine adherent versus non-adherent children revealed an increased rate of anti-infective prescriptions in non-adherent children but no difference for other outcomes.

We conclude that our results support the hypothesis that infection with measles is associated with long-term increased risk of other infectious diseases and that by preventing measles, vaccination is associated with non-specific heterologous improvements in health. However, because all of the non-measles controls received vaccination, we cannot rule out a direct benefit of vaccination to boost heterologous immune function, as has been suggested. ${ }^{25}{ }^{26}$ Nonetheless, the results fit with what would be expected from animal models and what has been shown in ecological studies and warrant further investigation into the long-term consequences of viral infections, particularly those with heightened tropism for immune memory cells on host resistance.

\section{Author affiliations}

${ }^{1}$ Department of Medical Informatics, Erasmus MC, Rotterdam, The Netherlands ${ }^{2}$ Department of Pathology, Harvard Medical School, Boston, Massachusetts, USA ${ }^{3}$ Department of Ecology and Evolutionary Biology, Princeton University, Guyot, New Jersey, USA

${ }^{4}$ Department of Viroscience, Erasmus MC, Rotterdam, The Netherlands

Contributors Conceived and designed the study: MJM, BG, DAMCvdV and RLDS. Code selection: KG, GMCM, DAMCvdV and RLDS. Data extraction and statistical analysis: CND, GMCM and MdR. Interpretation of data: all authors. Authored draft paper: KG and CND. Critical revisions of manuscript: KG, CND, GMCM, MdR, DAMCvdV, RLDS, MJM and BG. Study supervision: DAMCvdV, RLDS and MCJMS. Obtained funding: none.

Funding The authors have not declared a specific grant for this research from any funding agency in the public, commercial or not-for-profit sectors.

Competing interests None declared.

Patient consent Not required.

Ethics approval The study was approved by the independent THIN Scientific Review Committee (SRC reference number: 15-006).

Provenance and peer review Not commissioned; externally peer reviewed.

Data sharing statement № additional unpublished data from the study are available. All data are contained in the manuscript and the supplementary data files.

Open access This is an open access article distributed in accordance with the Creative Commons Attribution Non Commercial (CC BY-NC 4.0) license, which permits others to distribute, remix, adapt, build upon this work non-commercially, and license their derivative works on different terms, provided the original work is properly cited, appropriate credit is given, any changes made indicated, and the use is non-commercial. See: http://creativecommons.org/licenses/by-nc/4.0/.

\section{REFERENCES}

1. Rota PA, Moss WJ, Takeda M, et al. Measles. Nat Rev Dis Primers 2016;2:16049.

2. Strebel PM, Papania MJ, Fiebelkorn AP, et al. 20 - Measles vaccine A2 - Plotkin, Stanley A. In: Orenstein WA, Offit PA, eds. Vaccines. 6th edn. London: W.B. Saunders, 2013:352-87.
3. Griffin DE. Measles virus-induced suppression of immune responses. Immunol Rev 2010;236:176-89.

4. de Vries RD, de Swart RL. Measles immune suppression: functional impairment or numbers game? PLoS Pathog 2014;10:e1004482.

5. Simons E, Ferrari M, Fricks J, et al. Assessment of the 2010 global measles mortality reduction goal: results from a model of surveillance data. Lancet 2012;379:2173-8.

6. Aaby P, Bukh J, Lisse IM, et al. Measles vaccination and reduction in child mortality: a community study from Guinea-Bissau. J Infect 1984;8:13-21.

7. Allen IV, McQuaid S, Penalva R, et al. Macrophages and Dendritic Cells Are the Predominant Cells Infected in Measles in Humans. mSphere 2018;3:e00570-00517.

8. de Swart RL, Ludlow M, de Witte L, et al. Predominant infection of $\mathrm{CD} 150^{+}$lymphocytes and dendritic cells during measles virus infection of macaques. PLoS Pathog 2007;3:e178.

9. De Vries RD, McQuaid S, van Amerongen G, et al. Duprex WP and de Swart RL. Measles immune suppression: lessons from the macaque model. PLoS Pathog 2012;8:e1002885.

10. Laksono BM, de Vries RD, McQuaid S, et al. Measles Virus Host Invasion and Pathogenesis. Viruses 2016;8:e210:210.

11. Mina MJ, Metcalf CJ, de Swart RL, et al. Long-term measles-induced immunomodulation increases overall childhood infectious disease mortality. Science 2015;348:694-9.

12. IMS. Health UK: IMS Health Incorporated.2015 http://csdmruk. cegedim.com/.

13. Lewis JD, Schinnar R, Bilker WB, et al. Validation studies of the health improvement network (THIN) database for pharmacoepidemiology research. Pharmacoepidemiol Drug Saf 2007;16:393-401.

14. Bourke A, Dattani H, Robinson M. Feasibility study and methodology to create a quality-evaluated database of primary care data. Inform Prim Care 2004;12:171-7.

15. Blak BT, Thompson M, Dattani H, et al. Generalisability of The Health Improvement Network (THIN) database: demographics, chronic disease prevalence and mortality rates. Inform Prim Care 2011;19:251-5.

16. Booth N. What are the read codes? Health Lib Rev 1994;11:177-82.

17. Townsend $P$, Phillimore $P$, Beattie $A$. Health and deprivation. Inequality and the North. London: Chroom Helm Ltd, 1987.

18. Goldstein BA, Bhavsar NA, Phelan M, et al. Controlling for informed presence bias due to the number of health encounters in an electronic health record. Am J Epidemiol 2016;184:847-55.

19. UK Government Web Archive. Measles, Epidemiological data: 1996-2013. 2014 http://webarchive.nationalarchives.gov.uk/ 20140505192926/http://www.hpa.org.uk/Topics/InfectiousDiseases/ InfectionsAZ/Measles/EpidemiologicalData/ (updated 04 March 2014).

20. Janicke DM, Finney JW, Riley AW. Children's health care use: a prospective investigation of factors related to care-seeking. Med Care 2001;39:990-1001.

21. Freid VM, Makuc DM, Rooks RN. Ambulatory health care visits by children: principal diagnosis and place of visit. Vital and health statistics Series 13, Data from the National Health Survey 1998;137:1-23.

22. EU. Decision No. 2119/98/EC of the European parliament and of the council of 24 september 1998 setting up a network for the epidemiological surveillance and control of communicable diseases in the community. http://eur-lex.europa.eu/legal-content/EN/ALL/? uri=CELEX\%3A31998D2119.

23. Sonoda S, Nakayama T. Detection of measles virus genome in lymphocytes from asymptomatic healthy children. $J$ Med Virol 2001;65:381-7.

24. Sanders R, Dabbagh A, Featherstone D. Risk analysis for measles reintroduction after global certification of eradication. $J$ Infect Dis 2011;204:S71-S77.

25. Blok BA, Arts RJ, van Crevel R, et al. Trained innate immunity as underlying mechanism for the long-term, nonspecific effects of vaccines. J Leukoc Biol 2015;98:347-56.

26. Netea MG, Joosten LA, Latz E, et al. Trained immunity: a program of innate immune memory in health and disease. Science 2016;352:aaf1098. 